\title{
Article
}

\section{Crystalline 2D Covalent Organic Framework Membranes for High-Flux Organic Solvent Nanofiltration}

Digambar B. Shinde, Guan Sheng, Xiang Li, Mayur Ostwal, Abdul-Hamid Emwas, Kuo-Wei Huang, and Zhiping Lai

J. Am. Chem. Soc., Just Accepted Manuscript • DOI: 10.1021/jacs.8b08788 • Publication Date (Web): 05 Oct 2018

Downloaded from http://pubs.acs.org on October 9, 2018

\section{Just Accepted}

"Just Accepted" manuscripts have been peer-reviewed and accepted for publication. They are posted online prior to technical editing, formatting for publication and author proofing. The American Chemical Society provides "Just Accepted" as a service to the research community to expedite the dissemination of scientific material as soon as possible after acceptance. "Just Accepted" manuscripts appear in full in PDF format accompanied by an HTML abstract. "Just Accepted" manuscripts have been fully peer reviewed, but should not be considered the official version of record. They are citable by the Digital Object Identifier (DOI®). "Just Accepted" is an optional service offered to authors. Therefore, the "Just Accepted" Web site may not include all articles that will be published in the journal. After a manuscript is technically edited and formatted, it will be removed from the "Just Accepted" Web site and published as an ASAP article. Note that technical editing may introduce minor changes to the manuscript text and/or graphics which could affect content, and all legal disclaimers and ethical guidelines that apply to the journal pertain. ACS cannot be held responsible for errors or consequences arising from the use of information contained in these "Just Accepted" manuscripts. 


\section{INTRODUCTION}

In the chemical industry, organic solvent nanofiltration (OSN) employs membranes with pore size $1-2 \mathrm{~nm}$ to carry out concentration, exchange, purification, and recovery of organic solvents. ${ }^{1-2}$ It can also be used to implement novel processes such as membrane reactors. ${ }^{3}$ The demand for OSN grows steadily owing to stringent environmental laws and cost-effectiveness requirements. ${ }^{2}$ Compared to conventional separation technologies such as distillation, OSN is energy efficient, environment benign, conveniently scalable, and requires less foot-print. OSN is highly desirable for systems containing thermally sensitive species because the process does not require thermal energy.

The most effective approach to improve the membrane flux is to reduce the membrane thickness and/or increase the membranes' free volume. For example, Livingston and co-workers ${ }^{4}$ reported a novel interfacial polymerization method to prepare ultrathin polyamide membranes with thickness as low as $10 \mathrm{~nm}$, and the membrane exhibited unprecedented organic solvent fluxes. Chung and co-workers ${ }^{5}$ and Peinemann and co-workers ${ }^{6}$ reported the incorporation of nanoporous materials such as cyclodextrin into polymer matrixes to improve the membrane flux. However, most of these membranes, along with other polymer membranes reported in the field, have a dense and amorphous structure, which is not optimal for transport. Improving the flux of amorphous polymer membranes is inevitably compromised by loss in selectivity, or vice versa, which is the well-known permeability-selectivity trade-off issue of polymer membranes. ${ }^{7}$ Very recently, Liang and co-workers reported the synthesis of a conjugated microporous polymer (CMP) membrane. ${ }^{8}$ By increasing the porosity the membrane exhibited improved solvent fluxes for hexane and methanol.

Meanwhile, membranes made of pure ordered microporous materials such as zeolites and metal organic frameworks (MOFs) have exhibited superior performance to that of polymer membranes in gas separation and water desalination applications. ${ }^{9-13}$ However, although some ordered porous materials such MOFs have been used as fillers in mixed matrix membranes to improve the membrane performance in OSN applications, ${ }^{14-15}$ the full capability of ordered porous membranes has not been demonstrated yet. ${ }^{16}$ One reason is that most large-pore zeolites and MOFs exhibit inadequate structural and chemical stability in liquid separation. 2D COFs are a new class of ordered porous materials constructed through covalent bonds to form crystalline $2 \mathrm{D}$ porous structures. ${ }^{17-}$ ${ }^{21}$ Hence, 2D COFs have the most suitable microstructure for membrane applications. Most reported COFs have pore sizes of 1-2 nm. The covalent bonding render it stable in both aqueous solutions and organic solvents. ${ }^{22}$ Hence, 2D COFs are expected to be a highly potential type of membrane materials for OSN. However, the synthesis of continuous and defect-free COF membranes is still elusive, and reports of their applications in OSN are few. ${ }^{23-25}$

Langmuir-Blodgett (LB) is a potential method for preparing COF thin films of large dimensions. ${ }^{26}$ The LB method can precisely regulate the membrane thickness to the molecular scale. The obtained film can be transferred to different support surfaces. Using the LB 
method, we recently prepared an imine-bonded thin film and reported its application in nanofiltration for the first time. ${ }^{26}$ However, similar to other works ${ }^{27-28}$ the film was amorphous, and as a consequence, the water permeance was observed to be lower than 1 LMH/bar. Recently, Banerjee and co-workers reported COF membranes developed using solution casting and solvent-solvent interfacial polymerization methods. ${ }^{23-24}$ However, these membranes are significantly thick. Here, we report a novel synthesis strategy to prepare crystalline 2D COF membranes by the LB Method. The COF structure is formed through the $\beta$-ketoenamine linkage between the two precursors, 1,3,5-triformylphloroglucinol (TFP) and 9,9-dihexylfluorene-2,7-diamine (DHF), at the water-air interface. The membrane thickness is four-unit-cell. The flux of the obtained crystalline TPF-DHF membrane was over 100 times higher than that of our previously reported imine-bonded membrane ${ }^{26}$ and also higher than the best of the reported polymer membranes. ${ }^{4,8}$ Apparently, the well-defined porous structure accounts for the substantial improvement in performance; this clearly demonstrates the significant potential for the application of $2 \mathrm{D} \mathrm{COFs}$ as a new generation of membrane materials.

\section{EXPERIMENTAL SECTION}

Membrane preparation. The syntheses of TFP and DHF precursors are described in details in supplementary (section 1, ESI). The LB trough (Kibron MicroTroughX, Finland) was mounted on an anti-vibration table, and the whole setup was placed in a laminar flow hood to prevent contamination from dust. The trough and barriers are all made of Teflon. The surface pressure was measured by a Kibron Dyne Probe with a resolution of $1 \mu \mathrm{N} / \mathrm{m}$. Stock solutions of DHF and TFP were first prepared in toluene at concentration of $0.009 \mathrm{M}$ and $0.006 \mathrm{M}$, respectively. In a typical membrane preparation, the LB trough was first filled with water. $150 \mu \mathrm{L}$ of TFP and $150 \mu \mathrm{L}$ of DHF stock solutions were mixed together and then gently spread on the water surface using a micro syringe. After waiting for about $30 \mathrm{~min}$ to allow completely evaporation of toluene, the surface layer was compressed at a rate of $3.0 \mathrm{~mm} / \mathrm{min}$ till the surface pressure reached to $17 \mathrm{mN} / \mathrm{m}$. Then $500 \mu \mathrm{L}$ trifluoroacetic acid was added dropwise into the solution to initiate the reaction. The reaction was left undisturbed for $48 \mathrm{~h}$ at room temperature. After the completion of the reaction, a continuous and yellow TFP-DHF thin film was formed at the water-air interface. The transfer of the TFP-DHF thin film on support surface was conducted by either the Langmuir-Blodgett (vertical) method or the Langmuir-Schaefer (horizontal) method (Figure S1, ESI). In the first technique, the vertical support was lifted at a speed of $3.0 \mathrm{~mm} / \mathrm{min}$; whereas in the second technique, the horizontal support was lowered at a speed of $3.0 \mathrm{~mm} / \mathrm{min}$ till it was touched the water surface, and after waiting for a few seconds, the support was lifted at the same speed $(3.0$ $\mathrm{mm} / \mathrm{min}$ ) till it was detached completely from the surface.

Permeation studies. The membranes were mounted into a deadend filtration system with an effective membrane area of $2.54 \mathrm{~cm}^{2}$

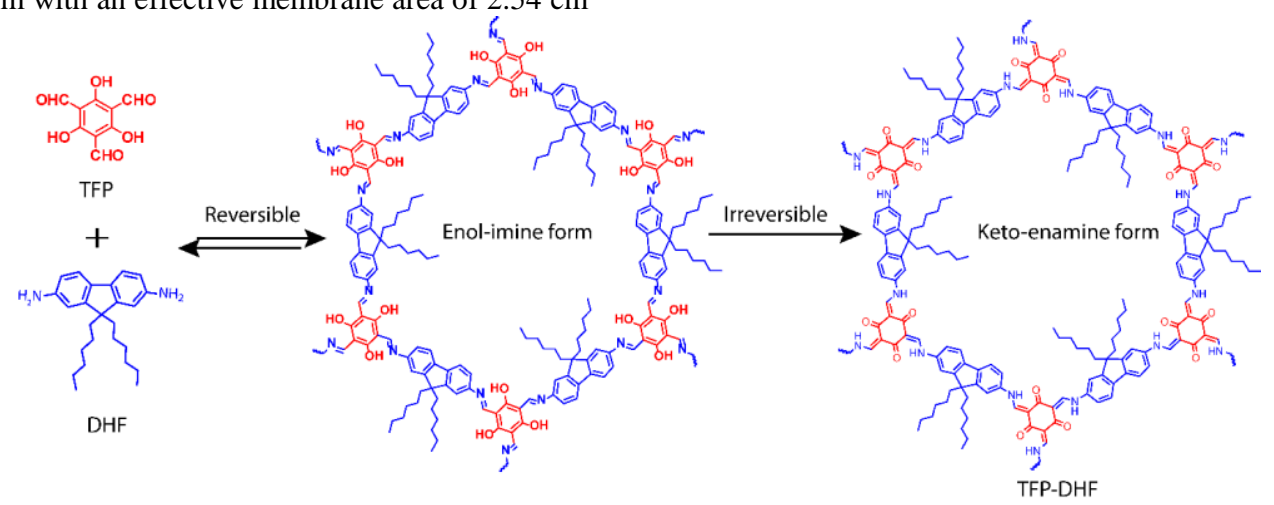

Scheme 1. TFP-DHF 2D COF thin film constructed by a one-pot two-step condensation reaction between 1,3,5-triformylphloroglucinol (TFP) and 9,9-dihexylfluorene-2,7-diamine (DHF).
(Figure S2, ESI). The measurements were conduct at room temperature and TMP of 1 bar. The solvent flux $(\mathrm{J})$ and permeance $(\mathrm{P})$ were calculated by the following equations,

$J=\frac{V}{A * t}$

$P=\frac{J}{\Delta p}$

Where; $V$ is the collected solvent volume (L) across the membrane during a time period of $\mathrm{t}(\mathrm{h}), \mathrm{A}$ is the effective membrane area $\left(\mathrm{m}^{2}\right)$, and $\Delta p$ is the trans-membrane pressure drop (bar). The dye rejection measurements were conduct in water and two organic solvents, methanol and ethanol. The feed concentration of the aqueous dye solutions is $50 \mathrm{ppm}$, but is $10 \mathrm{ppm}$ in organic solvents due to the low solubility of dyes in organic solvents., The rejection rate $(\mathrm{R})$ of dyes was calculated by the following equation:

$R=\left(1-\frac{C_{P}}{C_{F}}\right) \times 100 \%$

Where; $\mathrm{C}_{\mathrm{P}}$ and $\mathrm{C}_{\mathrm{F}}$ are the concentrations of dyes in the permeate and feed solutions, respectively. The concentration of dyes was measured by UV-Vis spectroscopy. To exclude the effect of solute adsorption, the concentration of the feed solution and permeate solution were analyzed when the permeation reached steady-state. All permeation processes reached steady-state within half an hour. Hence, the data were collected after 2 hours running and repeated on at least three samples. The average values were reported.

\section{RESULTS AND DISCUSSION}

The reaction between the two precursors, TFP and DHF, is illustrated in Scheme 1. Based on our previous experience, to form a thin film by the LB method, one precursor needs to be amphiphilic in order to form a monolayer assembled at the water-air interface. This was achieved by attaching two hexyl groups to 2,7-diaminofluorene to form DHF (Section 1, ESI). Both DHF and TFP are rigid and planar molecules, which aids the bond formation to occur along the well-defined directions to generate the required 2D hexagonal lattice. The condensation reaction between TFP and DHF involves two steps. The first step is a reversible Schiff-base condensation occurring between the amine group and the aldehyde group to generate the enol-imine structure. In the second step, an irreversible tautomerism reaction is occurred to form the $\beta$-ketoenamine structure. The first reversible step provides an error-correction mechanism to improve the structural crystallinity, whereas the second irreversible step helps to maintain the integrity of the framework structure. ${ }^{29}$ Furthermore, the condensation reaction can be tuned by acids, ${ }^{30-31}$ which permits better regulation of the reaction rate. 
During the synthesis, the LB trough was filled with water and placed on an anti-vibration table. The precursors, TFP and DHF, were first dissolved in toluene in 2:3 molar ratio and then dropcasted on the water surface. After toluene evaporated, the surface area was compressed by a Teflon barrier, and the surface-pressurearea isotherm was recorded. As shown in Figure 1a, when the surface area reduces, the surface pressure increases gradually. A small step was observed at approximately $17 \mathrm{mN} / \mathrm{m}$ surface pressure, which indicated the formation of a compact layer of precursors. The polymerization was initiated at this surface pressure by adding a catalytic amount of trifluroacetic acid (TFA), and the reaction was left undisturbed for $48 \mathrm{~h}$ at room temperature. After the completion of the reaction, a continuous and yellow TFP-DHF thin film was formed at the water-air interface. The obtained COF thin films were transferred to different support surfaces for different characterization purposes. The procedure can be repeated as many times as required to obtain membranes with different thickness. Figure 1b-d show the optical, SEM, and AFM images of a single layer
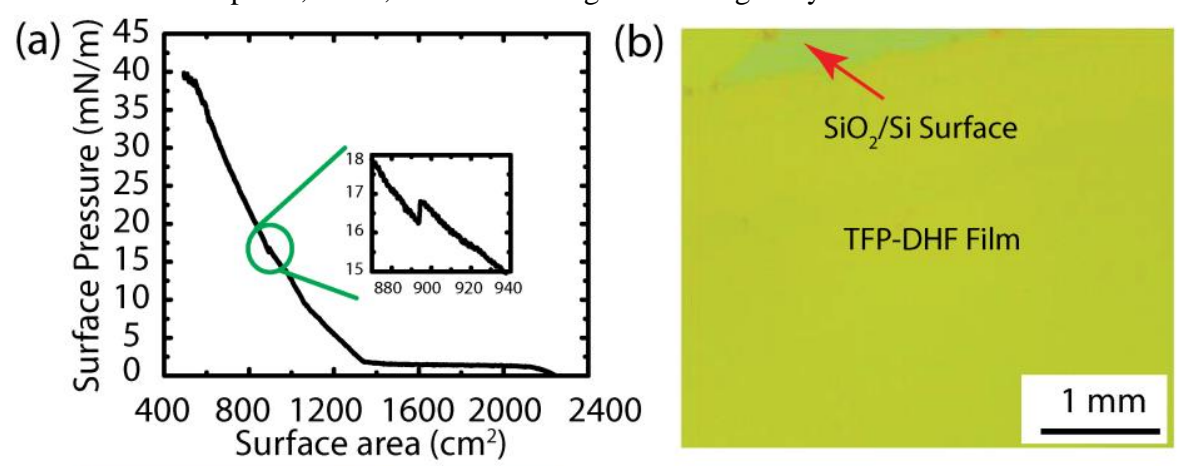

(c)

(d)
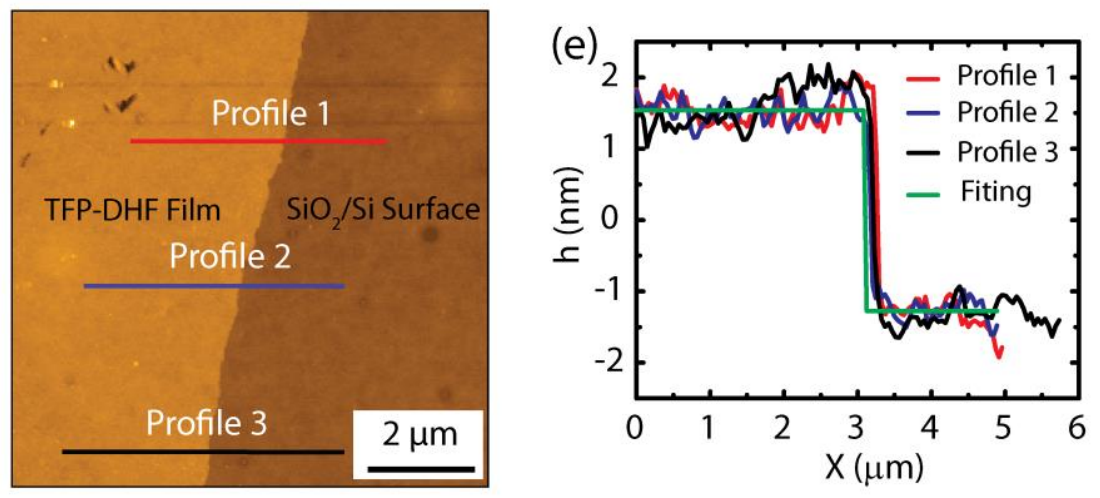

transferred to a smooth silica/silicon wafer. All images revealed that a continuous COF thin film was formed. The color contrast in the optical image (Figure 1b) is uniform, implying a uniform membrane thickness. Figure 1e shows three thickness profiles measured by AFM in the tapping mode along the scanning pathways that are illustrated in the AFM image in Figure 1d. The three profiles matched very well with each other, confirming again the uniform membrane thickness. The film thickness was approximately $2.9 \pm$ $0.3 \mathrm{~nm}$ with $0.5 \mathrm{~nm}$ roughness for a single layer. The thickness profiles measured from different samples (Figure S3, ESI) are also highly consistent, indicating that the membrane preparation procedure is well reproducible. Figure $1 \mathrm{f}$ shows the membrane thickness with respect to the number of coatings. A good linear relationship was obtained with a slope of $3.0 \mathrm{~nm}$, which agrees very well with the thickness of the single layer. Thus, the membrane thickness can be effectively regulated at the molecular level.

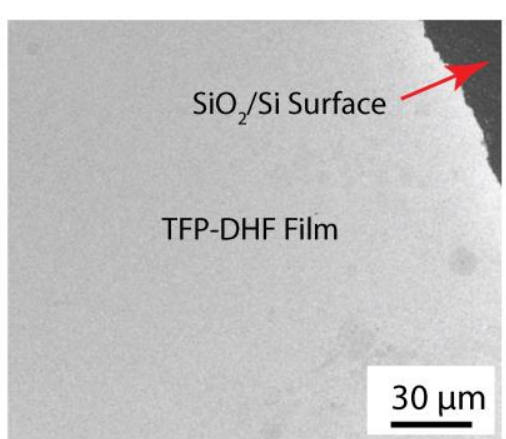

(f)

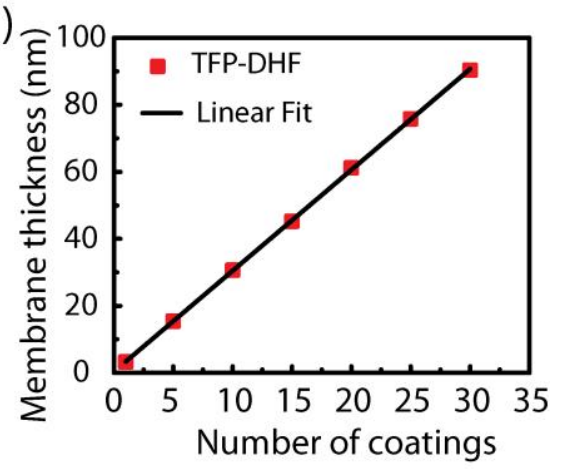

Figure 1. Membrane characterization (a) Surface-pressure-area isotherm, a step was observed at surface pressure of $17 \mathrm{mN} / \mathrm{m}$, and the polymerization was initiated at this surface pressure; (b) Optical microscopy image; (c) SEM image; (d) AFM topography image; (e) AFM height profiles of a single layer TFP-DHF film deposit on $\mathrm{SiO}_{2} / \mathrm{Si}$ surface, the AFM profiles were calculated using the Gwyddion software; and (f) Membrane thickness at different numbers of coatings

The structure of the COF thin film was determined by XRD analyses (Figure 2a). To obtain adequate signal intensity, the XRD pattern was recorded from a 30-layer COF film (M30). We also synthesized TFP-DHF micro-crystalline powder using the solvothermal method (section 2, ESI). The XRD patterns of the TFP-DHF COF film and the bulk powder were observed to be similar; both contain two strong peaks centered at approximately 5.04 and 12.68 and a broad peak in the range of $16.95-27.21$. The $2 \theta$ peaks appearing at 5.04 and 12.68 were indexed to be (100) and (001) planes, respectively. The broad peaks appearing in the range of $16.95-$ 27.21 corresponds to (002) plane. To elucidate the structure of the COF thin film, several stacking models were simulated (Figure S4, ESI). The XRD pattern was observed to be best matched with the inclined AA stacking model, as shown in Figure 2b. A Pawley refinement based on the film and the model XRD patterns gave the fitting parameters of $\mathrm{Rwp}=1.41 \%$ and $\mathrm{Rp}=1.74 \%$. After Pawley refinement, the unit cell parameters were obtained as follows: $a=$ $28.68 \AA, \mathrm{b}=28.49 \AA$, and $c=15.03 \AA ; \alpha=40^{\circ}, \beta=90^{\circ}$, and $\gamma=$ $120.14^{\circ}$ (Table S1, ESI). It can be seen that the dimension of a is very close to the value of $b$, and the angle between $a$ and $b$ is very close to $120^{\circ}$, so that the structure of each layer is close to a $2 \mathrm{D}$ hexagonal lattice, which implies that TFP and DHF formed a ordered hexagonal porous structure in each layer. The stacking of the COF layers is shifted, as illustrated in Figure 2c. The distance between adjacent layers was calculated approximately $7 \AA$. Thus, based on the film thickness measured by AFM, it can be concluded that each layer prepared by the LB method is not a monolayer, but 
comprises of 4 unit cells in thickness. The XRD peaks are broad, particularly for the (002) peak. This is most likely due to the incorporation of the alkyl chains, which disturb both the packing of layers and the size of unit cells. Similar effects have been observed in other COF structures with side chains. ${ }^{32-33}$
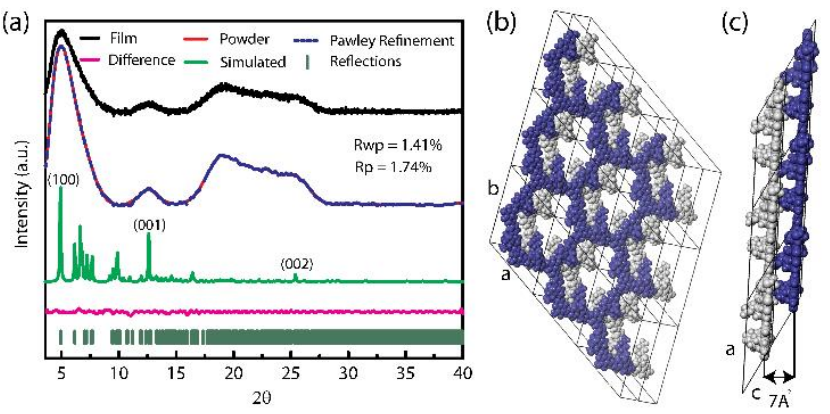

Figure 2. (a) XRD patterns and Pawley fitting. The film pattern was obtained from sample M30 (black), the powder XRD obtained from powder prepared by the solvothermal method (red), the simulated pattern obtained from the structure in (b) by the reflex module of Materials Studio ${ }^{\circledR}$ (green). The Pawley refinement, the observed reflections, and the difference plot are presented in blue, green, and pink, respectively. The Pawley fitting was also conducted by Materials Studio®. (b) A space-filled unit cell model generated by Materials Studio ${ }^{\circledR}$ illustrates the structure of the $\mathrm{COF}$ film in inclined AA stacking model. (c) Illustration of stacking shift between layers indicates that the average layer distance is approximately $7 \AA$.

The nitrogen adsorption isotherm of the TFP-DHF 2D COF is shown in Figure $3 \mathrm{a}$. The isotherm is a type-II pattern according to the IUPAC classification. The adsorption branch of the hysteresis loop is parallel to the desorption branch, which is a strong indication of a uniform pore size. ${ }^{34}$ From the adsorption isotherm, the BET surface area and the pore size were estimated to be $\sim 285 \mathrm{~m}^{2} / \mathrm{g}$ and $\sim 1.41 \mathrm{~nm}$ (Figure S5, ESI), respectively. The pore size estimated from the COF porous structure is around $1.28 \mathrm{~nm}$ (Figure S17, ESI), which is consistent to the pore size measured by BET. The relatively low surface area is due to the introduction of the hexyl chains because without the alkyl chain the surface area is around $365 \mathrm{~m}^{2} / \mathrm{g} .{ }^{35}$ The chemical bonding between the two precursors was characterized by FT-IR (Figure $3 \mathrm{~b}$ ) and Raman spectra (Figure S6, ESI). In the FT-IR spectra, the stretching bands of $\mathrm{CHO}$ and $-\mathrm{NH}_{2}$ groups, which appeared in the precursors, vanished completely in the film, indicating the complete consumption of the starting materials. The simultaneous appearance of new stretching frequency bands at $\sim 1606$ and $\sim 1578 \mathrm{~cm}^{-1}$ in the film correspond to $-\mathrm{C}=\mathrm{O}$ and $-\mathrm{C}=\mathrm{C}$ bonds, respectively, which indicate the formation of $\beta$-ketoenamine linkages in the COF film. In the Raman spectra of the film, the broad band in the range of $1612-1576 \mathrm{~cm}^{-1}$ correspond to the $-\mathrm{C}=\mathrm{O}$ and $-\mathrm{C}=\mathrm{C}$ groups, and the vibrational band at $1366 \mathrm{~cm}^{-1}$ correspond to aliphatic chains. The chemical structure was further surveyed by XPS analysis. The complete survey XPS plot of the TPF-DHF 2D COF film is shown in Figure 3c. Three intense peaks were observed in the spectra, which represent $\mathrm{C} 1 \mathrm{~s}$, $\mathrm{O} 1 \mathrm{~s}$, and $\mathrm{N} 1 \mathrm{~s}$ signals, respectively. In the high resolution spectra of $\mathrm{C} 1 \mathrm{~s}$ (Figure 3d), the two signals at $(284.78 \mathrm{eV})$ are for $\mathrm{C}=\mathrm{C}$ or $\mathrm{C}-$ $\mathrm{C}$, and the broad signal in the range $286.86-289.2 \mathrm{eV}$ was assigned to $\mathrm{C}-\mathrm{O}$ or $\mathrm{C}=\mathrm{O}$. Therefore, the signal at $284.78 \mathrm{eV}$ could be ascribed to the $\mathrm{C}=\mathrm{C}$ double bonds or $\mathrm{C}-\mathrm{C}$ bond present in the framework of TPF-DHF 2D COF. The high resolution N1s spectra (Figure $3 \mathrm{e}$ ) displayed only one strong signal at $400.29 \mathrm{eV}$, which corresponds to the enamine nitrogen $(\mathrm{C}=\mathrm{C}-\mathrm{NH}-)$ in the occurrence of the imine-enamine tautomerism process during the condensation reaction. Moreover, the signals at $531.28 \mathrm{eV}$ and $533.22 \mathrm{eV}$ could be attributed to the $\mathrm{C}=\mathrm{O}$ and $\mathrm{C}-\mathrm{O}$ bonds, respectively, displayed in the high resolution spectra of $\mathrm{O} 1 \mathrm{~s}$ (Figure $3 \mathrm{f}$ ). The formation of the $\beta$-ketoenamine linkage was further verified by ${ }^{13} \mathrm{C}$ cross polarization magic angle spinning (CP-MAS) (Figure S7, ESI), which revealed a characteristic signal at $\sim 184$ ppm corresponding to the carbonyl group along with the peaks in the range of $\sim 55-13$ ppm corresponding to alkylated chains. All the above results verified the formation of $\beta$-ketoenamine linkage in the TPF-DHF 2D COF framework. The thermogravimetric analyses (TGA) demonstrated that the TFP-DHF 2D COF is thermally stable up to $\sim 200^{\circ} \mathrm{C}$ (Figure S8, ESI). The contact angle of the TFP-DHF 2D COF film on an AAO disk was $84.5^{\circ}$, whereas the bare AAO disk yielded $27.5^{\circ}$ (Figure S9, ESI). The relatively hydrophobicity of the film should be partially owing to the hydrophobic alkyl chains.
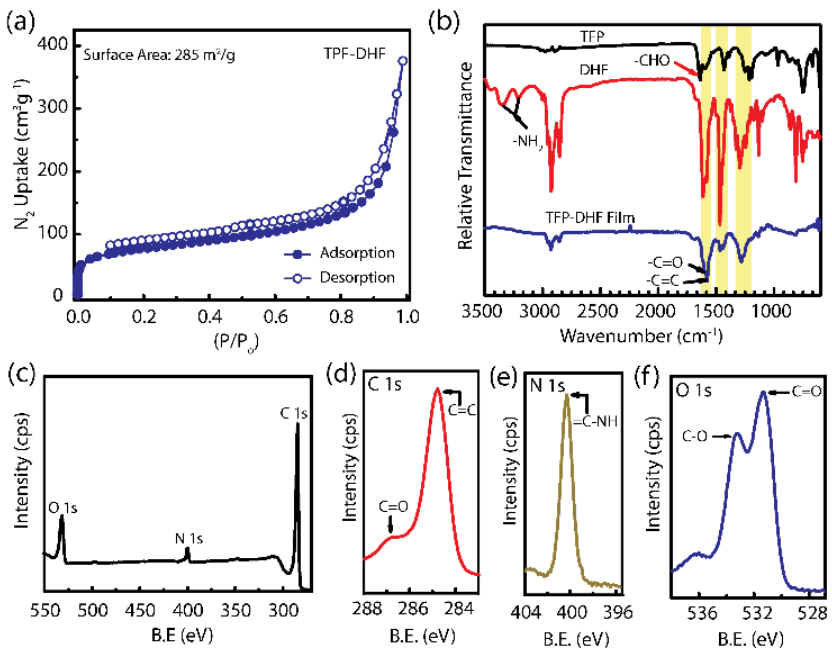

Figure 3. (a) Nitrogen adsorption isotherm of TFP-DHF 2D COF conducted at $77 \mathrm{~K}$; (b) FTIR spectra of TFP, DHF, and TFP-DHF 2D COF; (c) Typical XPS survey spectra of the TPF-DHF 2D COF film; (d, e, and f) the high resolution XPS spectra of $\mathrm{C} 1 \mathrm{~s}, \mathrm{~N} 1 \mathrm{~s}$, and $\mathrm{O} 1 \mathrm{~s}$, respectively.

Time evolutions of the membrane SEM, FT-IR and XRD were studied every $12 \mathrm{~h}$ in order to understand the membrane formation mechanism (Figure S18 and Figure S19, ESI). In the SEM images, small domains separated by interstitial gaps were observed in the first $12 \mathrm{~h}$. In the next $12 \mathrm{~h}$, almost all gaps were sealed, but grain boundaries could be observed clearly. Most of the grain boundaries disappeared after $36 \mathrm{~h}$ and a very homogeneous membrane was formed after $48 \mathrm{~h}$. The results of the FT-IR and XRD supported this progress growth model. The FT-IR spectra showed that after $12 \mathrm{~h}$ the starting materials were almost all consumed and the product signal remained almost constant after $12 \mathrm{~h}$. The XRD pattern showed almost no peak at $12 \mathrm{~h}$ and very broad peak at $24 \mathrm{~h}$ due to small crystalline domain. The peak become significant after $36 \mathrm{~h}$. Based on these results, Figure S19a illustrated the membrane formation procedure: the precursors first formed small domains; the domains then grew bigger and merged with each other; and finally a continuous and crystalline membrane was formed.

For permeation studies, we prepared three types of membranes, M15, M20, and M30, which correspond to 15, 20, and 30 numbers of layers, respectively, deposited on the AAO supports. The average pore size of the AAO support is $20 \mathrm{~nm}$. The cross-section SEM image of M20 is shown in Figure 4a. More cross-section and top view SEM images of membranes with different thicknesses were provided in Figure S11 and S10 in ESI. All of these images displayed that the COF membranes coated on top of the AAO supports 
were continuous with no penetration into the AAO pores. The thicknesses of M15, M20, and M30 are approximately 45, 61, and $90 \mathrm{~nm}$, respectively, which were measured from their SEM crosssection images (Figure S11, ESI). Figure 4b shows the permeances of water and various polar and non-polar organic solvents through the TFP-DHF 2D COF membrane at $25{ }^{\circ} \mathrm{C}$ under 1 bar transmembrane pressure drop (TMP) using a commercial dead-end filtration setup. The permeance is plotted with the reciprocal of the viscosity $(1 / \eta)$ of the solvents. A good linear relationship was observed. From the other hand, no direct relationship was observed between the membrane permeance and the molecular diameter of the solvents. It can be further seen from Figure $4 \mathrm{~b}$ that the permeance is inversely proportional to the membrane thickness. The linear relationship of the water flux with TMP is also verified in Figure S12 (ESI). We also run the permeation of water and various solvents up to $30 \mathrm{~h}$ (Figure S13, ESI) and in different pH (Figure S14 ESI). The permeance remained stable with no visible decline during the test period, which indicates the excellent structural and chemical stability of the TFP-DHF 2D COF membranes.
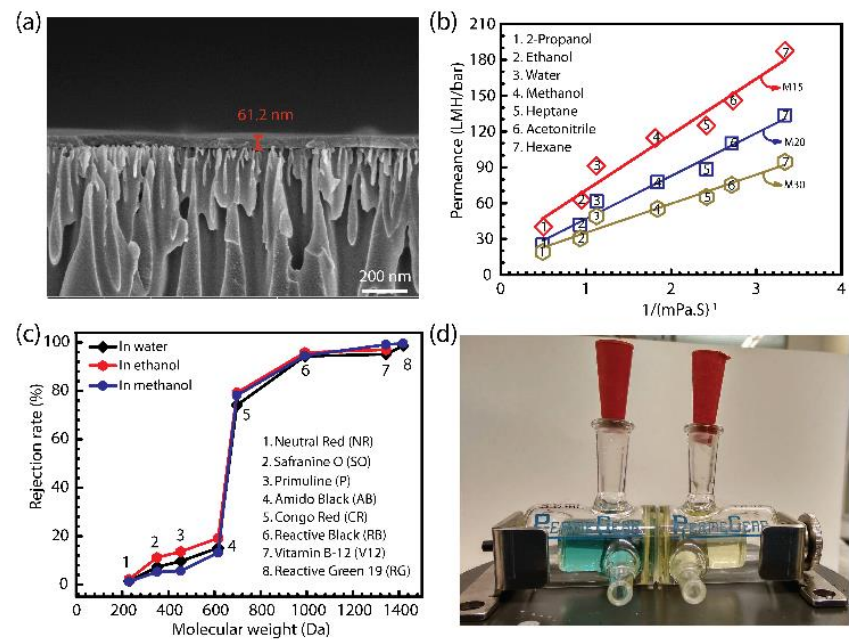

Figure 4: (a) Cross-section SEM image of sample M20; (b) Permeances of water and a number of polar and non-polar organic solvents through the three TFP-DHF 2D COF membranes with 15 (M15), 20 (M20), and 30 (M30) layers are plotted with the inverse of their viscosity; (c) Rejection rates of various dyes through the M20 membrane vs. their molecular weight; (d) Image showing the separation of the mixture dyes of Reactive Green (RG) and Primuline $(\mathrm{P})$. The chamber on the left-hand side contains the mixture of the two dyes, whereas the chamber on the right-hand side is filled with fresh water initially and turned yellow after 1-day diffusion.

To elucidate the transport mechanism, the simple Hagen-Poiseuille equation was used first to estimate the solvent permeance,

$P=\frac{n \pi d^{4}}{128 \eta L \tau}$

where $\mathrm{n}$ and $\mathrm{d}$ are the areal pore density and pore size, respectively, that can be estimated from the structure analysis (here, $2.1 \times 10^{17}$ and $1.41 \mathrm{~nm}$, respectively). Considering the water permeation data of the membrane with thickness $L=45.2 \mathrm{~nm}$, where $\eta$ (viscosity) $=8.9 \times 10^{-4} \mathrm{~Pa} \cdot \mathrm{s}, \mathrm{P}=91.3 \mathrm{LHM} / \mathrm{bar}$, we obtained the apparent tortuosity $\tau=1.33$. However, from the structure analysis the unit cell dimension along c-axis is $1.5 \mathrm{~nm}$, while the distance between two layers is $0.7 \mathrm{~nm}$, since the transport is along the c-axis, so without considering any other effects the tortuosity should be equal to 2.14. The smaller apparent tortuosity indicates that the non-slip boundary assumption used in the Hagen-Poiseuille equation is not valid and the slip boundary enhancement should be considered. This is consistent to the flows commonly observed in nanopores. ${ }^{36}$

Compared to our previously reported imine-bonded amorphous membranes prepared by the same LB process, ${ }^{26}$ the membrane thickness is equivalent, but the water permeance of the crystalline TFP-DHF 2D COF membrane is approximately 100 times higher. Compared to the permeation results of the best reported polymer membranes, ${ }^{4}$ we noticed a number of key differences. First, the permeability of the TFP-DHF 2D COF membrane is approximately 5 times higher than that of either the ultrathin polyamide membranes ${ }^{4}$ or the CMP membranes with rigid backbones. ${ }^{8}$ Considering that the pore sizes of the latter ones (MWCO $\sim 600 \mathrm{Da}$ ) are slightly smaller than the COF membrane (MWCO $900 \mathrm{Da}$ ), so the permeance of the COF membrane is at least equivalent to these bested performed polymer membranes. Secondly, the permeance of the TFP-DHF 2D COF membrane is proportional to $1 / \eta$, whereas that of the polyamide membrane is proportional to $\frac{\delta}{\eta d^{2}}$; here, $\delta$ is the solubility parameter, which reflects strong molecular interactions, and $\mathrm{d}$ is the molecular size of the solvents. Thirdly, the polyamide membrane requires pore activation; otherwise, the flux is significantly low. However, this is not required for the TFP-DHF 2D COF membrane. All of these differences can be explained by the structural difference, i.e., the well-ordered porous structure vs. the amorphous structure. The amorphous structure is dense with very low amount of permanent pores. Hence, it first requires an activation process to open the pores; secondly, the transport through dense polymer membranes is typically described by the solution-diffusion theory. The solubility of molecules in polymer can be analogous to the dissolution in liquid. Hence, the solubility parameter $\delta$ and the molecular size play an important role in membrane performance. Whereas the transport of porous membranes is governed by the pore-flow model. The transport is mainly related to solvent viscosity, which is a parameter mainly related to the solvent itself. Hence, the resistance through porous membranes is low and thus the permeance is high. All these results clearly indicate the structural merits of porous 2D COFs as building blocks for membrane applications.

The separation capability of the TFP-DHF 2D COF membrane (M20) was studied using the following dye molecules that have different molecular weight (size) in the range of the membrane pore size: natural red (NR) [MW 229; $0.87 \times 0.48 \mathrm{~nm}$ ], safranine O (SO) [MW 351; $0.91 \times 0.56 \mathrm{~nm}$ ], primuline $(\mathrm{P})[\mathrm{MW} 453 ; 1.02 \times 0.51$ nm], amido black (AB) [MW 616; $1.14 \times 0.78 \mathrm{~nm}$ ], congo red $(\mathrm{CR})$ [MW 696; $1.92 \times 0.87 \mathrm{~nm}$ ], reactive black $(\mathrm{RB})$ [MW 992; $1.65 \times$ $1.65 \mathrm{~nm}$ ], vitamin-B-12 (V12) [MW 1344; $1.8 \times 1.7 \mathrm{~nm}$ ], and reactive green $(\mathrm{RG})$ [MW 1419; $2.63 \times 1.97 \mathrm{~nm}]$. Among them, NR, V12 and RG are neutral charged, SO is positive charged, and P, $\mathrm{AB}, \mathrm{CR}$ and $\mathrm{RB}$ are negative charged. The rejections vs. the molecular weight in water, methanol and ethanol are plotted in Figure $4 \mathrm{c}$. The results in all three solvents are almost the same and all dyes showed a consistent $\mathrm{S}$-shaped rejection curve with molecular weight. Dyes can be rejected either by size exclusion and/or electrostatic repulsion. The zeta potential of the TFP-DHF membrane was measured to be around $-40 \mathrm{mV}$ at $\mathrm{pH} 7$, which indicated that the TFP-DHF membrane in principle could be more effective to reject negative dyes. However, considering that the studied dyes contain all type of charges, and also the fact that ionic dyes will not dissociate much in organic solvents, thus the effect of electrostatic repulsion can be largely excluded. Hence, the rejection should be mainly based on size exclusion. The separation capability is commonly defined by the molecular weight cut-off (MWCO) where the rejection rate is $90 \%$, while the steepness of size exclusion is defined by the molecular weight retention onset (MWRO) where the 
rejection rate is $10 \% .{ }^{37}$ From Figure $4 \mathrm{c}$, MWCO is read approximately as $900 \mathrm{Da}$ and MWRO as $600 \mathrm{Da}$. The difference between MWCO and MWRO of the TFP-DHF 2D COF membrane is much smaller than that of the CMP membranes, ${ }^{8}$ even though the latter one has a smaller MWCO. It is also much smaller than amorphous imine membrane prepared by the same method. Apparently, the very sharp molecular sieving of the COF membrane is the consequence of the well-ordered crystalline structure. Figure $\mathrm{S} 15$ showed a long-term rejection measurement of RG in aqueous solution for up to $10 \mathrm{~h}$. No penetration of RG through the membrane was observed during the studied time period. The water permeance decreased with time with an initial value close to the pure water permeance. Hence, the decline of water permeance with time is most likely due to accumulation of dye molecules on the membrane surface, owing to the dead-end permeation mode used. The separation of dye mixtures is further demonstrated in Figure 4d, in which the left chamber initially contained an aqueous solution of mixture dyes of RG and P in 1:1 molar ratio. The colors of RG and P are green and yellow, respectively, which imparts blue color to the mixture. The right chamber was initially filled with fresh water. After 1-day diffusion, the right chamber became yellow, indicating that only the small dye $\mathrm{P}$ passed through the membrane, whereas the big RG was blocked. This visual observation was confirmed by the UV-vis analysis (Figure S16, ESI). The UV-vis spectra of the feed solution contained two characteristic peaks at $342 \mathrm{~nm}$ and 635 $\mathrm{nm}$, which correspond to dye $\mathrm{P}$ and dye RG, respectively, whereas the UV-Vis spectra of the permeate solution contained only one peak at $342 \mathrm{~nm}$.

\section{CONCLUSION}

In summary, we demonstrated for the first time the synthesis of large-area crystalline 2D COF membranes by the LangmuirBlodgett method. The TFP-DHF 2D COF was constructed through $\beta$-ketoenamine linkages between TFP and DHF precursors, forming an inclined AA stacked hexagonal lattice with a pore size of approximately $1.41 \mathrm{~nm}$. A single layer prepared by the LB method is four-unit-cell thick, while the overall membrane thickness can be precisely regulated layer-by-layer. The membrane has a water contact angle of $84.5^{\circ}$, and was thermally stable up to $200{ }^{\circ} \mathrm{C}$. The well-defined porous structure of TFP-DHF 2D COF membrane significantly improved the solvent permeability by 100 times compared to those of the amorphous membranes prepared by the same process, and equivalent to those of the best reported polymer membranes. The permeation mechanism of the COF membrane was viscous flow with significant slip boundary enhancement, which is consistent to the flow through nanopores, but different from that of the polymer membranes. The membrane possesses permanent pores and does not require any pore activation. The TFP-DHF 2D COF membrane exhibited sharp molecular sieving with MWCO approximately $900 \mathrm{Da}$ and MWRO around $600 \mathrm{Da}$. All these results demonstrated the significant potential of 2D COF membranes for high-performance organic solvent nanofiltration applications.

\section{ASSOCIATED CONTENT}

Supporting Information. Synthesis details of linkers and COF, membrane preparation, permeation studies, characterization of membrane, simulation for theoretical COF structure. These details are provided in Supporting Information file. This material is available free of charge via the Internet at http://pubs.acs.org.

\section{AUTHOR INFORMATION}

\section{Corresponding Author}

*Zhiping.lai@kaust.edu.sa
ORCID

Zhiping Lai: 0000-0001-9555-6009

\section{Notes}

The authors declare no competing financial interest.

\section{ACKNOWLEDGMENT}

The work is supported by KAUST center competitive fund FCC/1/1972-19-01 and KAUST baseline fund BAS/1/1375-01-01.

\section{REFERENCES}

1. Vandezande, P.; Gevers, L. E. M.; Vankelecom, I. F. J., Solvent resistant nanofiltration: separating on a molecular level. Chem. Soc. Rev. 2008, 37 (2), 365-405.

2. Marchetti, P.; Jimenez Solomon, M. F.; Szekely, G.; Livingston, A. G., Molecular Separation with Organic Solvent Nanofiltration: A Critical Review. Chem. Rev. 2014, 114 (21), 10735-10806.

3. Lin, S.; Diercks, C. S.; Zhang, Y.-B.; Kornienko, N.; Nichols, E. M.; Zhao, Y.; Paris, A. R.; Kim, D.; Yang, P.; Yaghi, O. M.; Chang, C. J., Covalent organic frameworks comprising cobalt porphyrins for catalytic CO2 reduction in water. Science $\mathbf{2 0 1 5}$, 349 (6253), 1208-1213.

4. Karan, S.; Jiang, Z.; Livingston, A. G., Sub-10 nm polyamide nanofilms with ultrafast solvent transport for molecular separation. Science 2015, 348 (6241), 1347-1351.

5. Jiangtao, L.; Dan, H.; Yu, Z.; Susilo, J.; Tai-Shung, C., Precise Molecular Sieving Architectures with Janus Pathways for Both Polar and Nonpolar Molecules. Adv. Mater. 2018, 30 (11), 1705933.

6. Villalobos, L. F.; Huang, T.; Peinemann, K. V., Cyclodextrin Films with Fast Solvent Transport and Shape-Selective Permeability. Adv. Mater. 2017, 29 (26), 1606641.

7. Park, H. B.; Kamcev, J.; Robeson, L. M.; Elimelech, M.; Freeman, B. D., Maximizing the right stuff: The trade-off between membrane permeability and selectivity. Science $\mathbf{2 0 1 7}$, 356 (6343), 1138-1148.

8. Liang, B.; Wang, H.; Shi, X.; Shen, B.; He, X.; Ghazi, Z. A.; Khan, N. A.; Sin, H.; Khattak, A. M.; Li, L.; Tang, Z., Microporous membranes comprising conjugated polymers with rigid backbones enable ultrafast organic-solvent nanofiltration. Nat. Chem. 2018, 10 (9), 961-967.

9. Lai, Z. P.; Bonilla, G.; Diaz, I.; Nery, J. G.; Sujaoti, K.; Amat, M. A.; Kokkoli, E.; Terasaki, O.; Thompson, R. W.; Tsapatsis, M.; Vlachos, D. G., Microstructural Optimization of a Zeolite Membrane for Organic Vapor Separation. Science 2003, 300 (5618), 456-460.

10. Pan, Y. C.; Lai, Z. P., Sharp separation of C2/C3 hydrocarbon mixtures by zeolitic imidazolate framework-8 (ZIF-8) membranes synthesized in aqueous solutions. Chem. Commun. 2011, 47, 10275-10277.

11. Pan, Y. C.; Li, T.; Lestari, G.; Lai, Z. P., Effective separation of propylene/propane binary mixtures by ZIF-8 membranes. $J$. Membr. Sci. 2012, 390-391, 93-98.

12. Chen, W.; Chen, S.; Liang, T.; Zhang, Q.; Fan, Z.; Yin, H.; Huang, K.-W.; Zhang, X.; Lai, Z.; Sheng, P., High-flux water desalination with interfacial salt sieving effect in nanoporous carbon composite membranes. Nat. Nanotechnol. 2018, 13, 345-350.

13. Brown, A. J.; Brunelli, N. A.; Eum, K.; Rashidi, F.; Johnson, J. R.; Koros, W. J.; Jones, C. W.; Nair, S., Interfacial microfluidic 
processing of metal-organic framework hollow fiber membranes. Science 2014, 345 (6192), 72-75.

14. Campbell, J.; Burgal, J. D. S.; Szekely, G.; Davies, R. P.; Braddock, D. C.; Livingston, A., Hybrid polymer/MOF membranes for Organic Solvent Nanofiltration (OSN): Chemical modification and the quest for perfection. J. Membr. Sci. 2016, 503, 166-176.

15. Tham, H. M.; Japip, S.; Hua, D.; Chung, T.-S., Green Layerby-Layer Method for the Preparation of PolyacrylonitrileSupported Zinc Benzene-1,4-dicarboxylic Acid Membranes. ChemSusChem 2018, 11 (15), 2612-2619.

16. Echaide-Gorriz, C.; Sorribas, S.; Tellez, C.; Coronas, J., MOF nanoparticles of MIL-68(Al), MIL-101(Cr) and ZIF-11 for thin film nanocomposite organic solvent nanofiltration membranes. RSC Adv. 2016, 6 (93), 90417-90426.

17. Colson, J. W.; Woll, A. R.; Mukherjee, A.; Levendorf, M. P.; Spitler, E. L.; Shields, V. B.; Spencer, M. G.; Park, J.; Dichtel, W. R., Oriented 2D Covalent Organic Framework Thin Films on Single-Layer Graphene. Science 2011, 332 (6026), 228-231.

18. Ding, S.-Y.; Wang, W., Covalent organic frameworks (COFs): from design to applications. Chem. Soc. Rev. 2013, 42 (2), 548568.

19. Smith, B. J.; Parent, L. R.; Overholts, A. C.; Beaucage, P. A.; Bisbey, R. P.; Chavez, A. D.; Hwang, N.; Park, C.; Evans, A. M.; Gianneschi, N. C.; Dichtel, W. R., Colloidal Covalent Organic Frameworks. ACS Cent. Sci. 2017, 3 (1), 58-65.

20. Waller, P. J.; Gándara, F.; Yaghi, O. M., Chemistry of Covalent Organic Frameworks. Acc. Chem. Res. 2015, 48 (12), 30533063.

21. Côté, A. P.; Benin, A. I.; Ockwig, N. W.; Keeffe, M.; Matzger, A. J.; Yaghi, O. M., Porous, Crystalline, Covalent Organic Frameworks. Science 2005, 310 (5751), 1166.

22. Diercks, C. S.; Yaghi, O. M., The atom, the molecule, and the covalent organic framework. Science 2017, 355 (6328).

23. Kandambeth, S.; Biswal, B. P.; Chaudhari, H. D.; Rout, K. C.; Kunjattu, H. S.; Mitra, S.; Karak, S.; Das, A.; Mukherjee, R.; Kharul, U. K.; Banerjee, R., Selective Molecular Sieving in Self-Standing Porous Covalent-Organic-Framework Membranes. Adv. Mater. 2017, 29 (2), 1603945.

24. Dey, K.; Pal, M.; Rout, K. C.; Kunjattu H, S.; Das, A.; Mukherjee, R.; Kharul, U. K.; Banerjee, R., Selective Molecular Separation by Interfacially Crystallized Covalent Organic Framework Thin Films. J. Am. Chem. Soc. 2017, 139 (37), 13083-13091.

25. Matsumoto, M.; Valentino, L.; Stiehl, G. M.; Balch, H. B.; Corcos, A. R.; Wang, F.; Ralph, D. C.; Mariñas, B. J.; Dichtel, W. R., Lewis-Acid-Catalyzed Interfacial Polymerization of Covalent Organic Framework Films. Chem 2018, 4 (2), 308317.
26. Gadwal, I.; Sheng, G.; Thankamony, R. L.; Liu, Y.; Li, H.; Lai, Z. P., Synthesis of Sub-10 nm Two-Dimensional Covalent Organic Thin Film with Sharp Molecular Sieving Nanofiltration. ACS Appl. Mater. Interfaces 2018, 10 (15), 12295-12299.

27. Sakamoto, J.; van Heijst, J.; Lukin, O.; Schlüter, A. D., TwoDimensional Polymers: Just a Dream of Synthetic Chemists? Angew. Chem. Int. Ed. 2009, 48 (6), 1030-1069.

28. Dai, W.; Shao, F.; Szczerbiński, J.; McCaffrey, R.; Zenobi, R.; Jin, Y.; Schlüter, A. D.; Zhang, W., Synthesis of a TwoDimensional Covalent Organic Monolayer through Dynamic Imine Chemistry at the Air/Water Interface. Angew. Chem. Int. Ed. 2016, 55 (1), 213-217.

29. Kandambeth, S.; Mallick, A.; Lukose, B.; Mane, M. V.; Heine, T.; Banerjee, R., Construction of Crystalline 2D Covalent Organic Frameworks with Remarkable Chemical (Acid/Base) Stability via a Combined Reversible and Irreversible Route. $J$. Am. Chem. Soc. 2012, 134 (48), 19524-19527.

30. Segura, J. L.; Mancheño, M. J.; Zamora, F., Covalent organic frameworks based on Schiff-base chemistry: synthesis, properties and potential applications. Chem. Soc. Rev. 2016, 45 (20), 5635-5671.

31. Riddle, J. A.; Lathrop, S. P.; Bollinger, J. C.; Lee, D., Schiff Base Route to Stackable Pseudo-Triphenylenes: Stereoelectronic Control of Assembly and Luminescence. $J$. Am. Chem. Soc. 2006, 128 (34), 10986-10987.

32. Feldblyum, J. I.; McCreery, C. H.; Andrews, S. C.; Kurosawa, T.; Santos, E. J. G.; Duong, V.; Fang, L.; Ayzner, A. L.; Bao, Z., Few-Layer, Large-Area, 2D Covalent Organic Framework Semiconductor Thin Films. Chem. Commun. 2015, 51, 1389413897.

33. Tilford, R. W.; Mugavero, S. J.; Pellechia, P. J.; Lavigne, J. J., Tailoring Microporosity in Covalent Organic Frameworks. Adv. Mater. 2008, 20 (14), 2741-2746.

34. Do, D. D., Adsorption analysis: equilibria and kinetics. Imperial College Press: London, 1998.

35. Thote, J.; Barike Aiyappa, H.; Rahul Kumar, R.; Kandambeth, S.; Biswal, B. P.; Balaji Shinde, D.; Chaki Roy, N.; Banerjee, R., Constructing covalent organic frameworks in water via dynamic covalent bonding. IUCrJ 2016, 3 (Pt 6), 402-407.

36. Holt, J. K.; Park, H. G.; Wang, Y.; Stadermann, M.; Artyukhin, A. B.; Grigoropoulos, C. P.; Noy, A.; Bakajin, O., Fast Mass Transport Through Sub-2-Nanometer Carbon Nanotubes. Science 2006, 312, 1034-1037.

37. Ronco, C.; Clark, W. R., Haemodialysis membranes. Nat. Rev. Nephrology 2018, 14, 394-410.

\section{Table of Contents (TOC)}




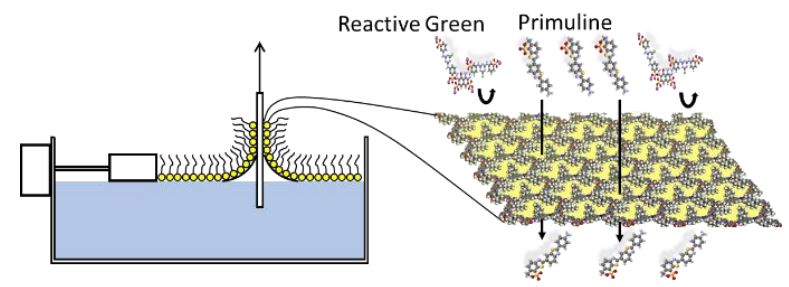

